Revista de Psicología de la PUCP. Vol. XVIII, 2, 2000

\title{
El abandono del tratamiento psiquiátrico: motivos y contexto institucional ${ }^{1}$
}

\author{
Alejandro Velázquez R. ${ }^{2} \quad$ Juan José Sánchez Sosa ${ }^{3}$ \\ Universidad Nacional Autónoma de México
}

\author{
María del Carmen Lara ${ }^{4}$ \\ Universidad Autónoma de Puebla
}

\author{
Héctor Sentíes 5 \\ Instituto Nacional de Psiquiatría
}

\begin{abstract}
Para documentar la permanencia en tratamiento de pacientes de una institución de salud mental y sus motivos de abandono, se analizaron 314 expedientes clínicos (177 mujeres y 137 hombres). Se contactó al total de pacientes localizables (64) en un análisis retrospectivo que cubrió siete años. Los resultados revelaron un porcentaje acumulativo de abandono del tratamiento superior a $70 \%$ sin tendencias atribuibles a edad, sexo o lugar de residencia. La categoría diagnóstica orgánico mostró un porcentaje de abandono ligeramente superior aunque inconsistente de año a año. Los motivos de abandono más frecuentes incluyeron los siguientes: sentirse mejor $(19.6 \%)$, atenderse en otra institución $(17.4 \%)$, falta de recursos económicos $(10.9 \%)$, motivos no tipificados $(7.6 \%)$, efectos colaterales de medicamentos prescritos $(6.5 \%)$ y maltrato del tratante $(6.5 \%)$. Se destaca la necesidad de diseñar e instrumentar intervenciones psicológicas para mejorar la atención y promover la adherencia terapéutica.
\end{abstract}

Palabras claves: consulta externa, tratamiento psiquiátrico, deserción, instituciones, México.

\section{Psychiatric outpatients dropout: Causes and institutional context}

This study examined treatment permanence of psychiatric outpatients, and identified reasons expressed by dropouts for discontinuing treatment. The clinical records of 314 patients (177 female and 137 male) were examined including a telephone follow-up on all former patients who could be found (64). The retrospective analysis spanned up to seven years after dropout. Results revealed a cumulative dropout percentage greater than $70 \%$ without identifiable trends attributable to patient gender, age, or place of residence. Diagnostic categories showed slightly higher but inconsistent dropout by "organic" patients. The most frequent reasons for stopping treatment included: feeling better $(19.6 \%)$, receiving treatment elsewhere $(17.4 \%)$, lacking financial resources $(10.9 \%)$, non-typified reasons $(7.6 \%)$, medication side effects $(6.5 \%)$, and caregiver rudeness $(6.5 \%)$. Results emphasize the need for effective psychological interventions designed to both improve institutional quality of treatment and promote treatment compliance. Key words: outpatients, psychiatric treatment, dropout, institutions, Mexico.

I El presente estudio constituyó un requisito parcial para la tesis profesional del primer autor.

2 Licenciado en Psicología. Ha colaborado con diversos proyectos de investigación del INP Ramón de la Fuente y en el Programa de Prevención Primaria de la División de Estudios de Posgrado de la UNAM. 

Los problemas de adaptación o funcionamiento psicológico constituyen una proporción significativa de la demanda de servicios de salud. Se estima que dichos problemas, expresados ya sea como dificultades de interacción con el ambiente (incluyendo el social o interpersonal) o como cuadros de aspecto somático, constituyen la tercera causa de consulta de primera ocasión en los servicios públicos de salud en México (Gutiérrez-Ávila y Barilar-Romero, 1986). En general, dichos servicios, sin embargo, se dan sólo a una pequeña proporción de las personas que los necesitan por dos razones principales. En primer lugar, la proporción de personas cuyos problemas de tipo psicológico muestran una severidad que amerite la búsqueda de ayuda profesional especializada es menor, en términos probabilísticos, que la que no los requiere. En segundo, aún cuando se busque un servicio especializado, el acceso a éste suele estar condicionado por múltiples factores entre los que podrían destacar los económicos, de localización geográfica y de cobertura institucional, entre otros.

En ocasiones, la severidad de los problemas psicológicos interfiere con el funcionamiento (individual, familiar, laboral o comunitario) a grado tal que la persona requiere atención especializada (Buckley, 1994). En estos casos, las opciones de servicio suelen buscarse en función del acceso del individuo o la familia a servicios de salud públicos o privados determinados por su capacidad económica

3 Profesor e investigador. Obtuvo la maestría y doctorado en psicología y desarrollo humano en la Universidad de Kansas. Preside la International Society of Clinical Psychology y coordina la Red Internacional de Salud como vicepresidente de la IUPsyS. Correo electrónico: johannes@servidor.unam.mx.

4 Médico psiquiatra por la UNAM. Profesora titular, investigadora y coordinadora del Departamento de Investigación en Psiquiatría de la facultad de medicina de la UAP.

5 Médico psiquiatra por la UNAM. Investigador y jefe de Consulta Externa de Servicios Clínicos del INP Ramón de la Fuente. 
Alejandro Velázquez R., Juan José Sánchez Sosa, María del Carmen Lara y Héctor Sentíes

o por una cobertura de servicios de salud previstas como prestación laboral del usuario mismo o de algún familiar inmediato.

Entre los servicios especializados en salud destacan los prestados por institutos de carácter nacional. Estos institutos suelen hacer un énfasis particular en la noción de que la calidad de un servicio de salud depende en buena porción de su derivación a partir de resultados de investigación. Así, estos institutos fungen simultáneamente como centros de tratamiento y de investigación científica.

En el caso de la salud mental en México, el Instituto Nacional de Psiquiatría "Ramón de la Fuente" reúne, en efecto, ambas funciones o características. Por una parte desarrolla proyectos de investigación que van desde el nivel neuro y psicofisiológico hasta el epidemiológico y social y, por otra, ofrece servicios de tratamiento psiquiátrico buscando en ambos casos mantenerse en los mejores niveles de calidad sin más límite que el determinado por los recursos disponibles.

En general se busca que un tratamiento restaure la funcionalidad del individuo y de quienes le rodean. Este restablecimiento suele depender de series complejas de factores en constante interacción que van desde antecedentes interpersonales, hereditarios o clínicos del individuo y su familia, hasta la aplicación sistemática de procedimientos terapéuticos bien diseñados o seleccionados. Estos procedimientos suelen, a su vez, incluir intervenciones que van desde el uso prescrito de medicamentos hasta la aplicación de intervenciones psicoterapéuticas de muy diversa índole.

Generalmente la planeación e instrumentación de intervenciones terapéuticas se basa en el supuesto de que un tratamiento surte efecto sólo cuando se le instrumenta con atingencia y continuidad sin interrumpirse hasta que los indicadores de restablecimiento y funcionalidad del paciente ameriten darle de alta. Así, el abandono de un tratamiento supone, no sólo un desperdicio de recursos, sino un riesgo adicional de 
El abandono del tratamiento psiquiátrico: motivos y contexto institucional

recaídas que pueden llevar a disfuncionalidades progresivamente más incapacitantes.

En el contexto de optimización los recursos existentes en los servicios de salud, tanto en países "desarrollados" como en los de economía "en transición" se ha observado que los recursos resultan insuficientes si su aplicación no se estudia y vigila cuidadosamente. En países como México, un porcentaje importante de la población no está cubierta por los servicios de salud y aquellos que lo están, no necesariamente se benefician de ellos. La explicación de esto es compleja y de origen multifactorial; asimismo, en México, tal como ocurre en el resto del mundo, los pacientes no siempre cumplen o se apegan a su programa terapéutico. Una de las modalidades de esta carencia de adherencia terapéutica es el abandono o deserción de un tratamiento.

Esta falta de cumplimiento o adherencia al tratamiento es un problema serio para cualquier componente del sector salud. Si se analizan los recursos utilizados, desde los materiales hasta los salarios del personal esto se hace evidente. Un paciente psiquiátrico a quien se atiende en una institución es recibido por una recepcionista quien toma sus datos; una secretaria que abre un expediente; una enfermera que lo recibe y lo lleva a la evaluación correspondiente que puede incluir estudios de laboratorio y gabinete, y finalmente, personal médico y de psicología que instrumenta las intervenciones apropiadas al tratamiento, todo a costa de importantes recursos salariales a los que se suman los de infraestructura, equipo, mantenimiento, etc.

Una vez prescrito un tratamiento se informa o instruye al paciente o su familia sobre el régimen, y la responsabilidad de llevarlo a cabo recae casi exclusivamente en éstos. Con frecuencia esto puede ocurrir sin que el paciente tenga una información y conciencia clara de su padecimiento y las destrezas mínimas para instrumentarlo. $\mathrm{La}$ información incluye las implicaciones y consecuencias, ya sea de 
continuar el tratamiento o de abandonarlo (Pang, Tso, Ungvari, Chiu y Leung, 1995; Swanson, Pantalon y Cohen, 1999).

La literatura de investigación reciente contiene algunos ejemplos de la forma en que el abandono de un tratamiento o su conducción parcial repercute en perjuicio del restablecimiento del paciente. En general, se asume que una tasa de abandono mayor a $30 \%$ es alta; algunos estudios informan tasas de deserción consistentemente superiores, especialmente en pacientes con problemas de adicción (Ross, Cutler y Sklar, 1997; Siqueland et al., 1998), aunque también se han documentado programas terapéuticos con una muy alta retención de pacientes aún en esta área (Wiseman, Henderson y Briggs, 1997).

Algunos predictores de abandono del tratamiento presentados por literatura especializada (Grilo et al., 1998) han incluido factores tales como bajo ingreso familiar, actitudes negativas del paciente hacia el tratamiento, atribución de crisis de angustia a estresores externos y edad avanzada. Con menor valor predictivo se han incluido las siguientes: baja escolaridad, tratamientos previos de corta duración, hipersensibilidad a la angustia, baja evitación agorafóbica y búsqueda de apoyo social como estilo predominante de afrontamiento

Un estudio exploratorio con pacientes que dejaron de asistir a sus citas en la consulta externa del instituto reveló que la frecuencia de deserción en un periodo de tres meses en 1993 fue de $7 \%$ y las principales causas aparentes del abandono incluyeron lo siguiente: (a) falta de recursos para comprar el medicamento (15\%); (b) recibir atención médica en otro sitio (12\%); y (c) no haber observado ninguna mejoría a corto plazo ( $11 \%)$. El diagnóstico que prevalecía en estos pacientes era el de trastornos afectivos. El estudio concluyó que las causas de deserción estaban relacionadas con el paciente mismo y no con la calidad de la atención que otorga el instituto (Saucedo, 1997). En este estudio, sin embargo, sólo se realizó un seguimiento a corto plazo con una muestra relativamente pequeña. 
Dentro de la investigación de la adherencia o permanencia terapéutica de pacientes psiquiátricos también conviene examinar la influencia de los medicamentos. Recientemente se ha incrementado la efectividad de estos tratamientos de modo muy notable. Se ha evidenciado, por otra parte, que para muchos cuadros clínicos dichos fármacos no logran los resultados esperados, lo cual puede convertirse en motivo de abandono del tratamiento (Zimbroff, Kane y Tamminga, 1998). Es también frecuente que estos fármacos produzcan efectos secundarios, que pueden ser sumamente molestos (Hoencamp, Haffmans y Duivenvoorden, 1998).

En ocasiones los medicamentos sólo modifican algunos síntomas del padecimiento a condición de mantenerse casi permanentemente con dosis de sostén. Hay casos en que el paciente debe permanecer en un seguimiento sujeto a controles periódicos que pueden resultar molestos y precipitar el abandono del tratamiento ante la creencia de que la mejoría alcanzada se mantendrá con o sin fármacos. Esta suerte de rebelión a las medidas prescritas puede conducir a recaídas o complicaciones generándose así un circulo vicioso (Pucheu, 1984).

Un modelo de investigación que ha ayudado a la comprensión de los padecimientos mentales es el modelo médico-psiquiátrico el cual ha logrado evolucionar de la unicausalidad a la multicausalidad aceptando la intervención de factores biológicos, psicológicos y sociales. Con alguna frecuencia se emplea el modelo para la explicación de prácticamente todos los cuadros clínicos a pesar de que se corre el riesgo de considerar a los factores psicosociales como si fueran de la misma naturaleza que los biológicos. Así, por ejemplo, se puede privilegiar el efecto de medicamentos al suponer que éste se extenderá a los aspectos de interacción interpersonal del paciente. Si bien puede haber algún efecto en este sentido vía la inducción química de cambios en reactividad emocional, no es esperable que reestructure la adaptabilidad derivada de habilidades interactivas por lo cual se requieren también intervenciones de habilitación (Bedell, Lennox, Smith y Rabinowicz, 1998). 
Se han documentado algunas asociaciones entre la permanencia en tratamiento y factores de muy diversa naturaleza tal como el tipo de terapia utilizada en pacientes psiquiátricos (Herinckx, Kinney, Clarke y Poulson, 1997), historia de abuso de alcohol y funcionamiento social deficiente (Hoencamp et al., 1998), escolaridad (Siqueland et al., 1998), somatización (Ross et al., 1997), la relación terapeuta-paciente (Clinton, 1996) y desempleo y edad (Tehrani, Krussel, Borg y Munk-Jorgensen, 1996), entre otros.

En principio, si los orígenes etiológicos de cualquier padecimiento psicológico suelen incluir la interacción de componentes fisicoquímicos, de habilidades de afrontamiento de las demandas del ambiente (incluyendo el interpersonal) y de competencia social, no es esperable que la conducta de adherirse a un régimen terapéutico ocurra en función de variables predominantemente biológicas (Piper, Joyce, McCallum y Azim, 1998). Así, el análisis de los componentes que influyen sobre dicha adherencia se convierte en un objeto de estudio legítimo y necesario.

En efecto, en la medida en que la falta de adherencia terapéutica es un problema serio que enfrenta el subsector de la salud mental, se hace necesario iniciar exploraciones más sistemáticas de los motivos que parecen predecir el abandono de un tratamiento por parte de los pacientes. Así, el objetivo del presente estudio consiste en explorar la posible relación de variables tales como sexo, edad, lugar de residencia, diagnóstico clínico y personalidad, con la permanencia de un tratamiento o la deserción del mismo. Una adición metodológica de la presente investigación consistió en instrumentar un seguimiento telefónico de pacientes que interrumpieron su tratamiento en periodos que oscilaron de los seis meses a los seis años. 


\section{Metodología}

La presente investigación se puede clasificar en varias dimensiones lógicas y metodológicas generalmente aceptadas al respecto sus propósitos, tipo de recolección de datos y naturaleza de los análisis realizados (Plutchik, 1974). Se trata de un estudio retrospectivo en virtud de haber recolectado una porción importante de los datos extrayéndolos de los expedientes clínicos de los pacientes, atendidos por primera vez en el año de 1993. Es a su vez un estudio de corte longitudinal en el sentido de haberse realizado un seguimiento a siete años desde la primera asistencia a consulta en el Instituto y el carácter de su permanecía hasta el año 1999.

Se trata también de un estudio exploratorio en cuanto su propósito es la identificación de las condiciones en las que ocurre un fenómeno más que el análisis de la contribución relativa de componentes específicos a valores particulares de su ocurrencia. Es un estudio de campo en el sentido de que la porción principal de los datos sobre la variable independiente se originaron en las localidades de residencia de los participantes.

Finalmente, tuvo componentes de estudio de gabinete en cuanto que la captura de los datos sobre el resto de las variables se realizó en el área de servicios clínicos del Instituto Mexicano de Psiquiatría y el procedimiento de jueceo para la validación de las categorías de motivos de abandono del tratamiento en las instalaciones de la División de Estudios de Posgrado de la Facultad de Psicología de la Universidad Nacional Autónoma de México.

\section{Participantes}

La selección de la muestra fue aleatoria y todos los registros de consulta externa en el año en cuestión tuvieron la misma oportunidad de resultar seleccionados. La muestra estuvo compuesta por 314 expedientes clínicos de un total de 3323 registrados; éstos fueron 
clasificados en la consulta externa del instituto y pertenecieron al periodo del 1 de enero al 31 de diciembre de 1993, con un seguimiento de siete años (1999). Los expedientes presentaban un registro básico, pero no todos estaban completos pues algunos incluian registros de pacientes enviados sólo a estudios de laboratorio. A una primera muestra aleatoria de cincuenta registros que arrojó 36 expedientes clínicos y 14 estudios de laboratorio, se añadió una segunda que se acercara a un mínimo de $10 \%$ de expedientes clínicos. Se tomaron 450 registros adicionales que con los 50 anteriores dieron un total de 500 (15.03\%). De estos, $314(9.44 \%)$ eran expedientes clínicos propiamente dichos y 186 $(5.59 \%)$ estudios de laboratorio excluidos del análisis final.

Del total, 177 (56\%) eran mujeres y $137(44 \%)$ hombres, entre los 13 y 91 años de edad. En cuanto al lugar de residencia, 267 (85\%) provinieron de la Ciudad de México y 47 (15\%) de otros estados de la república; todos fueron atendidos por causas relacionadas con un diagnostico clínico y/o de personalidad.

La muestra de 314 pacientes fue contactada vía telefónica. De ésta, $273(86.95 \%)$ dejaron de acudir a sus citas programadas antes de 1999. Los 41 (13.05\%) pacientes restantes se distribuyen en 33 $(10.50 \%)$ que continuaron su tratamiento hasta el año de 1999,7 $(2.22 \%)$ a quienes se dio de alta o dio "cita abierta" y uno (0.33\%) se canalizó a otra institución.

\section{Variables}

Como variable dependiente se tomó la ausencia de registro de asistencia a consulta a partir de la última a la que asistió cada paciente en conjunción con la expectativa específica de continuación de tratamiento; es decir, la ausencia de un juicio clínico-institucional de haber sido dado de alta o haberse concluido el tratamiento.

La variable independiente fue la expresión verbal registrada en relación al motivo del abandono del tratamiento dada por el paciente mismo o por un familiar inmediato en caso de indisponibilidad del 
primero, ante la pregunta ¿cuál fue el motivo por el cual dejo de acudir a Consulta en el Instituto?

Como variables adicionales para el análisis, recolectadas de los expedientes clínicos se incluyeron las siguientes: (a) Edad: la edad cronológica de cada paciente al momento de la fecha de la primera consulta, tal y como aparece registrada en su expediente; (b) Sexo: el género masculino o femenino al cual pertenece cada paciente, registrado en su expediente; (c) Lugar de residencia: el lugar geográfico en el cual habita o habitaba el paciente durante el tiempo que acudió a tratamiento; (d) Diagnóstico clínico: el estado del cuadro sintomático asignado por el equipo de salud especializado, en función de la clasificación diagnóstica, bajo los lineamientos de los sistemas DSM-III o DSM-IIIR (los vigentes al momento de la primera consulta); (e) Personalidad: las características más estables y duraderas a partir de los juicios clínicos específicos hechos al respecto, contenidas en todas las notas hechas por el equipo de salud y congruentes con los criterios DSM-IV, clasificados por grupos de personalidad: A, B, C, orgánica o sin diagnóstico (First, Spitzer, Gibbon y Williams, 1995).

\section{Instrumentos}

1. El expediente clínico incluía los siguientes datos:

- Motivo de consulta.

- Problemas o padecimiento actual.

- Interrogatorio para valorar aparatos y sistemas.

- Situación familiar, laboral, social y económica.

- Antecedentes personales.

- Consumo de substancias tóxicas y problemas relacionados.

- Antecedentes familiares.

- Examen del estado mental.

- Temperamento y carácter.

- Examen físico general y neurológico. 
- Examen y tratamientos previos.

- Exámenes (en su caso) de laboratorio y gabinete.

- Observaciones generales.

- Diagnóstico.

- Indicaciones terapéuticas y recomendaciones.

- Evaluación del tratamiento.

- Fecha.

- Firma del médico.

\section{Cédula de deserción}

Validez. Antes de definir la clasificación que serviría de base para la captura informática definitiva de los datos de motivos de abandono del tratamiento y su análisis se realizó un estudio de validación por expertos, de las posibles categorías a que podrían pertenecer dichos motivos.

Una vez capturadas transcribiéndolas verbatim (tal como las había dicho el participante entrevistado) el total de las transcripciones integras de las entrevistas telefónicas se vaciaron a una hoja denominada Cédula de deserción. En una primera clasificación se enlistaron las categorías a que podían pertenecer los motivos de abandono del tratamiento dados por los participantes y se pidió a doce jueces, diez estudiantes avanzados de psicología clínica y dos profesores de la misma especialidad de la facultad de psicología de la Universidad Nacional Autónoma de México que, después de leer cada motivo de abandono, lo asignaran a la categoría que le correspondiera de acuerdo con su juicio personal, en la cédula correspondiente. Cada juez trabajó en esta tarea con completa independencia y sin comunicación con los demás jueces o con los conductores del jueceo.

Si un motivo de abandono no resultaba asignado a una categoría específica por un mínimo de $80 \%$ de los juicios independientes de los jueces, la redacción que definía a la categoría se refinaba y se volvía a pasar por el procedimiento de jueceo hasta que $80 \%$ o más 
de los motivos cayeran en una categoría. Si en una segunda vuelta de jueceo una categoría de la cédula no mostraba una consistencia interjueces mayor al $80 \%$ los motivos en cuestión pasaban a la categoría otros motivos. Sólo un $1.08 \%$ del total de los motivos registrados cayó en dicha categoría y ningún motivo de abandono requirió más de dos vueltas por el procedimiento de jueces para lograr el criterio mínimo de consistencia. Finalmente se registraron quince categorías para los motivos de abandono del tratamiento.

\section{Procedimiento}

Una vez concluida la captura y examen de los datos de los 314 expedientes seleccionados aleatoriamente, de pacientes atendidos por primera vez en 1993, los datos correspondientes a la hoja de vaciado se trasladaron a una base de datos computarizada Fox Plus. A partir de estos datos se separaron a los pacientes en dos grupos: uno de los desertores y uno de pacientes que continuaban asistiendo a consulta hasta el año de 1999.

Los pacientes que desertaron de su consulta antes de 1999 incluyeron un total acumulativo de $273(86.95 \%)$, a los cuales se contactó vía telefónica. De esos, a 209 (66.57\%) resultó imposible localizarlos por diversas causas tales como que el paciente no vivía en el teléfono registrado, el número telefónico no existía, la familia del paciente no disponía de servicio telefónico, no lo conocían en el domicilio contactado, etc. Se logró localizar a los 64 pacientes $(20.38 \%)$ restantes quienes contestaron a la pregunta estándar: ¿Cuál fue el motivo por el cual dejó de acudir a consulta externa en el Instituto? El investigador anotaba textualmente las respuestas de los participantes lo cual arrojó un total de 92 causas de abandono distintas, pues hubo pacientes que refirieron más de una razón para haber dejado de asistir a sus consultas. 
Alejandro Velázquez R., Juan José Sánchez Sosa, María del Carmen Lara y Héctor Sentíes

\section{Resultados}

Las Figuras 1, 2 y 3 presentan datos de los años 1993 a 1998. Los del año 1999 (10.50\%) no se incluyen por tratarse de los pacientes que continuaban en tratamiento durante ese año, al momento de la realización del estudio.

La Figura 1 muestra la distribución porcentual de abandono del tratamiento a lo largo de los seis años cubiertos en el estudio, y añade la representación del abandono por edad a fin de inspeccionar posibles fluctuaciones conjuntas. Durante los primeros dos años (1993 y 1994), el porcentaje de pacientes que abandonó el tratamiento fue dos y tres veces mayor ( $19.4 \%$ y $39.5 \%$, respectivamente) a prácticamente todos los datos desde 1995 hasta 1998. En éstos últimos, el porcentaje de abandono se estabilizó alrededor de una mediana de 7.7\%. Excepto por la disminución general en las tasas de abandono, los datos no denotan ninguna otra tendencia identificable. La información sobre la edad promedio de los pacientes muestra una distribución relativamente plana en torno a los 38 años. Esta tendencia se ratifica en la distribución de la edad del total de pacientes entre 22 y 40 años (Figura 3). Los valores oscilaron entre un mínimo de $1.6 \%$ y un máximo de $4.8 \%$ de pacientes en cualquier categoría de edad, sin mostrar una tendencia identificable al alza o a la baja. Los porcentajes de pacientes menores de 22 años y mayores de 40 es mínima, derivándose incluso de frecuencias menores a cinco pacientes.

La Figura 2 representa la distribución porcentual de los pacientes que abandonaron el tratamiento en función del tipo de personalidad anotado por el médico en las entrevistas iniciales, por año, a lo largo de los seis abarcados en el estudio. La distribución de porcentajes no denota tendencias identificables en el sentido de asociarse consistentemente con alguno de los tipos de personalidad asignados, a excepción de tres de las seis medidas pertenecientes a cada año $(1996,1997$ y 1998), donde los porcentajes más altos de deseción fueron de pacientes clasificados como orgánicos. Esta misma categoría, sin embargo mostró porcentajes muy 
El abandono del tratamiento psiquiátrico: motivos y contexto institucional

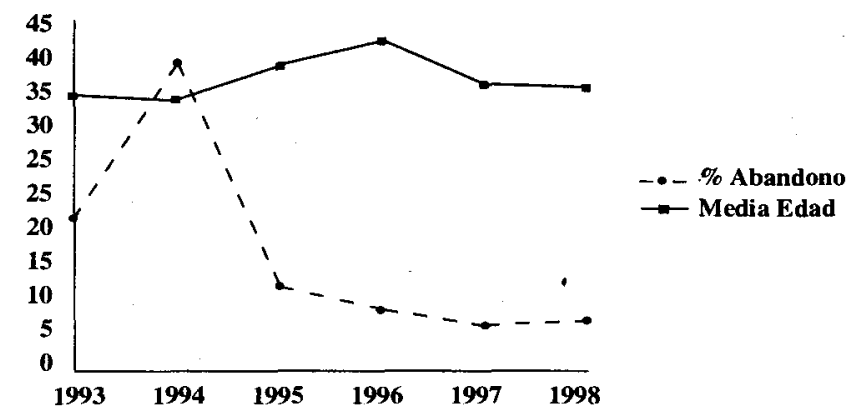

Figura 1. Porcentaje de abandono del tratamiento psiquiátrico distribuido por año y promedio de edad.

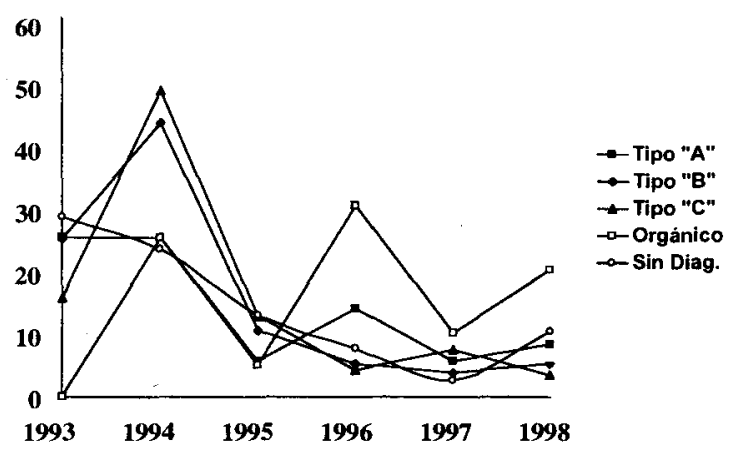

Figura 2. Porcentaje de abandono del tratamiento psiquiátrico distribuido por año y tipo de personalidad.

cercanos o idénticos a otros tipos de personalidad en otros tres años y en uno (1993) arrojó la más baja de todos. En uno de los tres años en que resultó un porcentaje alto (1997), no superó en más de $2.6 \%$ al tipo de personalidad que siguió en el orden (Tipo C).

Finalmente, la Figura 3 muestra los porcentajes relativos a los motivos aducidos por los respondientes para la suspensión indefinida o abandono del tratamiento provisto en la consulta externa del Instituto. 
En orden descendiente, las razones más frecuentes incluyeron haber sentido mejoría (19.56\%), haberse atendido en otra institución $(17.39 \%)$, falta de recursos $(10.86 \%)$, problemas diversos no tipificados no asignables a los otros motivos $(7.60 \%)$, molestias o efectos colaterales de los fármacos prescritos $(6.52 \%)$ y maltrato por parte del médico $(6.52 \%)$. El resto de los motivos, con menos de $5.5 \%$ incluyeron lo siguiente: fallecimiento, haberse resuelto el problema por el que acudió a consulta, habérsele dado de alta, no vivir en la Ciudad de México, problemas administrativos varios, la lejanía de las instalaciones del servicio de salud, el no desear el tratamiento prescrito, haberse suicidado y una categoría general de otros motivos no tipificables, los dos últimos con porcentajes menores a $2 \%$.

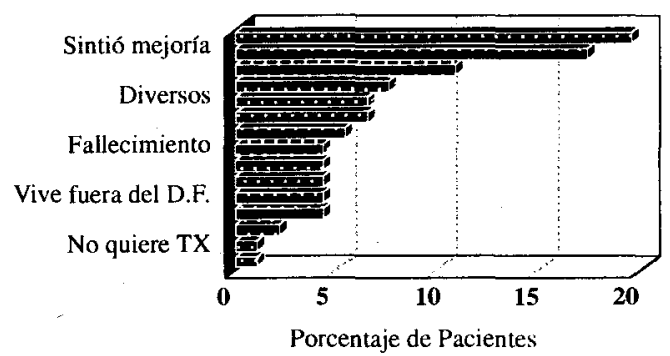

Figura 3. Motivos principales de abandono del tratamiento psiquiátrico.

Los porcentajes de pacientes desertores del tratamiento por año, en función del sexo de éstos mostró que, excepto en 1993 -en que el total de hombres que desertó fue casi un tercio más que las mujeresy 1994 -cuando el total de mujeres que abandonó el tratamiento superó a los hombres en una proporción similar- la distribución arrojó diferencias mínimas, menores a 5\% en los años. Al igual que en el caso de las distribuciones por edad, los únicos datos atípicos se concentraron en los dos años iniciales en que los porcentajes de abandono del tratamiento fueron particularmente altos, especialmente en 1994. De los cinco años en que los datos de deserción de hombres y mujeres 
arrojaron alguna diferencia, en tres hay más desertores y en dos más desertoras.

Las distribuciones porcentuales de pacientes que abandonaron su tratamiento en función del sitio o área geográfica en que viven o vivían al momento de su primera consulta, no revelaron consistencia respecto a variaciones asociadas a la distancia geográfica hasta el Instituto. En sólo dos de los seis años: 1996 y 1998, se dio una relación en ese sentido. Sin embargo otros años mostraron relaciones que no ratificaron el sentido (1995 y 1997); asimismo, en un año (1993) desertaron más pacientes residentes en la ciudad de México que otros que residen en el Estado de México o en lugares más distantes del interior.

La distribución porcentual de diagnósticos clínicos en que resultaron clasificados los pacientes en su primera consulta no mostraron tendencia alguna al respecto del abandono del tratamiento. Es probable que la diversidad de diagnósticos presentados haya arrojado porcentajes de comparación demasiado pequeños para hacerlos claros y definidos. Los trastornos del estado de ánimo conformados por el de depresión mayor $(23.89 \%)$, distimia (10.51\%), depresión doble $(2.87 \%)$ y el trastorno bipolar $(2.55 \%)$ representaron conjuntamente un $39.82 \%$, y resultaron los padecimientos con mayor frecuencia dentro de la demanda del servicio. En siguiente lugar se dieron los trastornos esquizofrénicos $(12.42 \%)$, ataques de pánico $(8.92 \%)$, síndrome orgánico cerebral $(6.36 \%)$, reacción de ajuste $(6.36 \%)$ y otros que recibieron algún tipo tratamiento pero no un diagnóstico formal (4.14\%). Los restantes correspondieron a trastornos del dormir, trastorno de angustia generalizada, trastorno limítrofe, síndrome demencial, crisis parciales de sintomatología compleja y otros diagnósticos, ninguno con más de un $3 \%$ de prevalencia. 


\section{Discusión}

El estudio tuvo como propósito documentar la permanencia de los pacientes de una institución psiquiátrica en sus citas programadas en consulta externa y los motivos referidos por los que abandonaron su tratamiento. Se añadió una exploración descriptiva de características de los pacientes tales como edad, sexo, lugar de residencia, personalidad y diagnóstico clínico, cotejándolas con el abandono y realizando un seguimiento que osciló de uno a siete años dependiendo de la última fecha de consulta registrada.

En los servicios de salud mental se espera que los pacientes atendidos dejen de acudir a estos sólo cuando el experto tratante lo indica por alta, cita abierta o alguna otra expresamente ponderada por el equipo de salud. Se espera que estos pacientes se mantengan a mediano y largo plazo en tratamiento debido a que, en ocasiones, los padecimientos psiquiátricos suelen permanecer durante toda la vida. Sin embargo, ésto no ocurre debido aparentemente a que no basta dar indicaciones al paciente o a su familia -lo cual a veces se hace rutinariamente- para continuar un tratamiento

En principio esto significaría que ni la información ni una conciencia clara del padecimiento son condiciones suficientes para mantener al paciente desempeñando las acciones inherentes a su tratamiento. Entre el primero y el segundo año de asistir a tratamiento un 58.92\% de los pacientes lo abandonan. En un seguimiento a siete años el abandono se acumula dejando una media de sólo $10.51 \%$ de permanencia en tratamiento en el séptimo año. De esta manera, parece claro que estos padecimientos requieren intervenciones de mantenimiento que aumenten la permanencia de los pacientes en su tratamiento.

La edad de los pacientes que acuden a este servicio reveló una media de 38 años aproximadamente. Los pacientes que conforman el grupo de desertores estudiado muestra un promedio de edad de 22 a 40 años conformando un $56.2 \%$ del total del continuo de edad. Estas 
edades son claves debido a que mucha de la vida productiva del hombre ocurre dentro de este promedio de edad. Así, además del malestar individual y familiar que entraña un problema psiquiátrico, el abandono del tratamiento implica consecuencias negativas también en ámbitos como el laboral y el de una vida comunitaria activa. En cuanto a género, en contraste con la suposición de que los hombres abandonan mas frecuentemente el tratamiento que las mujeres, las proporciones difieren en poca medida.

El lugar de residencia es un factor que en ocasiones se ha asociado con la permanencia de los pacientes en su tratamiento. En principio se supondría que a menor distancia se encuentre el servicio de salud del domicilio del paciente mayor consistencia y apego presentaría éste respecto de su tratamiento. Los hallazgos del presente estudio, sin embargo, mostraron que la deserción se da en proporciones aproximadamente iguales en aquellos pacientes que viven dentro de la ciudad de México y aquellos que radican en el interior. La demanda de atención es básicamente de la población que vive en la ciudad $(85.03 \%)$ donde se encuentran las instalaciones del servicio. Hay una demanda del $8.2 \%$ de pacientes que radican en el Estado de México y de $6.68 \%$ de aquellos que se encuentran en otros estados de la república mexicaná. Es posible que este contraste entre el tamaño de la demanda local y la foránea en el sentido de porcentajes muy dispares obscurezca un posible efecto de la distancia geográfica.

Los diagnósticos con los cuales se clasifica a estos pacientes frecuentemente incluyen los trastornos de personalidad. Estos no suelen ser formas aisladas de psicopatología, sino que suelen encontrarse en co-morbilidad con otros síndromes clínicos. El hecho de que haya desacuerdo sobre la importancia diagnóstica de los componentes de los cuadros co-mórbidos dificulta la comprensión de su efecto sobre el abandono del tratamiento. Los resultados sobre los tipos de personalidad asignados a los pacientes revelaron una mayor prevalencia de deserción en aquellos que pertenecen al tipo orgánico (por ejemplo retardo mental). Dicho efecto no es, sin embargo, ni consistente ni 
de gran monta. Los tipos B y C de personalidad también presentaron un índice relativamente alto de deserción.

La salud mental es un fenómeno determinado por factores tan complejos como los sociales, ambientales, biológicos y psicológicos, entre otros. La recuperación de un estado funcional depende, en parte, de una instrumentación eficaz de esfuerzos terapéuticos orientados a detectar y controlar trastornos considerados epidemiológica y funcionalmente como importantes, tales como la depresión, la ansiedad, la esquizofrenia y las demencias. A su vez, la instrumentación exitosa de servicios de salud mental depende en parte del grado de confiabilidad con que se conozcan los tipos de padecimientos y su frecuencia relativa. En el presente estudio, los trastornos del estado de ánimo resultaron los más comunes. Lo anterior concuerda con los hallazgos de Saucedo (1997) que indicaron que en la consulta externa de la División de Servicios Clínicos del instituto los pacientes atendidos con mayor frecuencia son los que presentan trastornos afectivos (del estado de ánimo). Asimismo concuérdan con los hallazgos de la World Health Organization (1996) que revelaron que los trastornos más relevantes en esta área, desde el punto de vista de la salud pública incluyen los afectivos (bipolar, manía, depresión y distimia), los trastornos de ansiedad (crisis de angustia, trastorno obsesivo-compulsivo y estrés post-traumático) seguidos de la esquizofrenia y las demencias.

Desde la óptica de la medicina, De la Fuente (1982) ha propuesto que un aspecto de la crisis actual de la especialidad es que el tipo de atención que hoy en día se ofrece se aleja cada vez más de lo que los usuarios potenciales esperan. Un hecho verificable es que muchos pacientes no se adhieren al régimen prescrito por el equipo de salud ya sea porque no comprenden lo que el médico les dice o porque desconfían de sus actitudes o razonamientos. Muchos médicos parecen percibir la realidad clínica casi exclusivamente en términos de explicaciones biológicas a las que consideran como definitorias y como las únicas reales y clínicamente significativas. Esto relega a otras explicaciones y a las reacciones personales de los pacientes a un plano que pareciera trivial. 
El abandono del tratamiento psiquiátrico: motivos y contexto institucional

Sin embargo, los resultados sobre motivos del abandono del tratamiento documentados en el presente estudio sugieren que numerosos factores psicológicos, entrelazados con su nexo familiar, social y cultural, juegan un papel importante en la evolución de muchos problemas médicos afectando las respuestas al tratamiento. Las interpretaciones que hacen los pacientes acerca del motivo por el cual dejaron el tratamiento bosqueja un panorama más claro de las causas o factores que contribuyen al abandono, con un nivel razonable de credibilidad y se esperaría que tuvieran algún impacto a nivel institucional. El conocimiento de dichos motivos referidos por los pacientes permitiría guiar el diseño y la instrumentación de tratamientos más inclusivos y efectivos y de estrategias para promover la permanencia en aquéllos.

El motivo sintió mejoría podría estar ligado con lo mencionado por Zimbroff et al. (1998) acerca de la efectividad cada vez mayor de los medicamentos de desarrollo reciente. El efecto de los medicamentos incluye aspectos subjetivos de la enfermedad ya que la sintomatología corregida con ellos no está necesariamente relacionada con un bienestar real en cuanto funcionalidad. Con el tiempo esto se refleja en recaídas o deterioro en otras esferas de los pacientes. Es posible que los pacientes, al sentirse mejor, decidan que no hace falta continuar el tratamiento o que el efecto del medicamento no ha mejorado áreas de su funcionamiento laboral o familiar de la manera que mejoraron aspectos emocionales.

Los resultados sobre el motivo se atiende en otra institución puede reflejar, como también ha propuesto De la Fuente (1982), que cuando no se cumplen las expectativas de tratamiento existe poca adherencia a éste. Muchos pacientes pueden haber decidido probar suerte en otra institución cuyo personal o equipo de salud reunía, a su juicio, características más cercanas a esa expectativa. En este ámbito, sería muy importante incorporar a las rutinas de recepción, diagnóstico y tratamiento de los pacientes, aspectos de interación interpersonal y destrezas de comunicación verbal y no verbal orientadas a identificar dichas expectativas y ajustar, en lo posible los componentes pertinentes 
Alejandro Velázquez R., Juan José Sánchez Sosa, María del Carmen Lara y Héctor Sentíes

del tratamiento, ésto por parte de todos los integrantes del equipo de salud.

Los resultados sobre el motivo por falta de recursos incluiría no sólo altos costos de los medicamentos prescritos sino también los de traslado al instituto, el costo de la consulta y de estudios requeridos, entre otros. En contraste con los hallazgos de Saucedo (1997), éste no parece ser el principal factor de deserción ya que más bien parece tratarse de un claro efecto multifactorial. Por ejemplo, el motivo problemas diversos incluye aquellos relativos a un contexto social del individuo que abarca desde aspectos familiares hasta problemas de concepción del tratamiento, o un simple permiso para faltar al trabajo. En virtud de que la responsabilidad de llevar a cabo el tratamiento recae en gran parte sobre la familia y sobre otros miembros de la comunidad inmediata del paciente, los servicios de salud deberían incluir intervenciones sistemáticas a fin de que los familiares realmente estén en posibilidad de apoyar el proceso terapéutico.

El motivo por las molestias del medicamento, como refieren Hoencamp et al. (1998), incluye todas aquellas que producen efectos secundarios molestos, los cuales, relacionados quizá con la poca información recibida del médico sobre esos efectos y formas de mitigarlos pueden generar temor o reticencia en el paciente. Este temor puede originarse en la interpretación catastrófica del paciente sobre sensaciones que desconoce o que le son muy poco familiares. Nuevamente, la intervención del psicólogo con estrategias de reestructuración cognoscitiva en trabajo multidisciplinario contribuiría a reducir dichos efectos.

Los hallazgos en la categoría por maltrato del médico entraña aspectos delicados pues la discusión de este tema incluye factores complejos. Se comparte la opinión de estudios como el de Clinton (1996) que indican que entre mejor sea la relación médico-paciente será más viable un mejor apego al tratamiento. Sería conveniente abordar este problema desde, por lo menos, dos perspectivas. Por una 
parte, los programas de formación de psiquiatras se verían beneficiados con la adición de contenidos y prácticas relativos al mejoramiento de la relación médico-paciente. Por otra, la inclusión sistemática del psicólogo como coterapéuta que instrumente intervenciones (ya evaluadas y documentadas en la literatura de investigación) para mejorar tanto el funcionamiento del paciente como su apego al tratamiento farmacológico.

Es evidente que en un estudio de este tipo, el abandono del tratamiento por fallecimiento sirvan principalmente como un dato descriptivo, excepto en casos de suicidio plausiblemente atribuibles al problema psiquiátrico mismo y que en el presente estudio reveló proporciones mínimas (un paciente). Por ultimo los motivos referidos con un porcentaje menor a un 5\% incluyeron lo siguiente: los que resolvieron el problema que los llevo a consulta, los que refirieron haber sido dados de alta (que probablemente mintieron ya que en su expediente no existe ninguna nota en ese sentido). Factores como vivir fuera del DF (y por la distancia), haber experimentado problemas administrativos (como errores de calendarización) y no querer tratamiento, tuvieron los porcentajes más bajos excepto "otros" en que incurrió sólo un paciente que dijo seguir asistiendo a tratamiento normalmente confirmándose no haber sido el caso.

Aunque se podría concluir que variables como edad, sexo, lugar de residencia, diagnóstico clínico y personalidad, muestran una relación poco clara con el abandono del tratamiento éstas, en efecto, facilitan la comprensión de este fenómeno de gran complejidad. Todo parece indicar que no bastan las variables tipificadas en el presente estudio para explicarlo a toda satisfacción. Los motivos expuestos por los pacientes abren nuevas perspectivas para el estudio más pormenorizado de la deserción clínica.

La continuación de esta línea de investigación en diversos contextos institucionales latinoamericanos muy probablemente se beneficiaría si se valoran cualitativa y cuantitativamente datos como la motivación 
hacia el tratamiento, las expectativas del paciente, su disponibilidad de tiempo y recursos, entre otras. También se deberán buscar muestras cuyo tamaño y tipificación clínica mejoren su carácter de representativos tanto en sentido estadístico como social o clínico. También convendría incluir variables cognoscitivas como la tolerancia a la frustración, resistencias y dinámica familiar. Una línea lógica de continuación de esta línea de investigación incluiría el realizar réplicas sistemáticas (Sidman, 1960) considerando metodologías y observaciones que permitan un seguimiento más sólido.

\section{Referencias}

Bedell, J., Lennox, S. S., Smith, A. D. y Rabinowicz, E. F. (1998). Evaluation of problem solving and communication skills of persons with schizophrenia. Psychiatry Research, 78(3), 197-206.

Buckley, R. A. (1994). Differentiating medial and psychiatric illness. Psychiatric Annals, 24(11), 584-591.

Clinton, D. N. (1996). Why do eating disorder patients drop out? Psychotherapy and Psychosomatics, 65(1), 29-35.

De la Fuente, R. (1982). Factores socioculturales en la situación clínica. Salud Mental, 5(1), 1-2.

First, M. B., Spitzer, R. L., Gibbon, M. y Williams, J. (1995). The structured clinical interview for DSM III-R personality disorders (SCID-II): I. Description. Journal of Personality Disorders, 9(2), 83-91.

Grilo, C. M., Money, R., Barlow, D. H., Goddard, A. W., Gorman, J. M., Hofmann, S. G., Papp, L. A., Shear, M. K. y Woods, S. W. (1998). Pretreatment patient factors predicting attrition for multicenter randomized controlled treatment study for panic disorder. Comprehensive Psychiatry, 39(6), 323-332.

Gutiérrez-Ávila, J. H. y Barilar-Romero, E. (1986). Morbilidad psiquiátrica en el primer nivel de atención en la Ciudad de México. Boletín de la Oficina Sanitaria Panamericana, 101(6), 648-657. 
El abandono del tratamiento psiquiátrico: motivos y contexto institucional

Herinckx, H. A., Kinney, R. F., Clarke, G. N. y Paulson, R. I. (1997). Psychiatric Services, 48(10), 1297-1306.

Hoencamp, E., Haffmans, P. M. J. y Duivenvoorden, H. J. (1998).

Predictability of dropout in unipolar depressed patients. European Psychiatry, 13(2), 63-66.

Pang, A. H., Tso, S., Ungvari, G. S., Chiu, H. y Leung, C. M. (1995). An audit study of defaulters of regular psychiatric outpatient appointments in Hong Kong. International Journal of Social Psychiatry, 41(2), 103-107.

Piper, W. E., Joyce, A. S., McCallum, M. y Azim, H. F. (1998). Journal of Consulting and Clinical Psychology, 66(3), 558-567.

Plutchik, R. (1974). Foundations of experimental research. Nueva York: Harper \& Row.

Pucheu, C. (1984). Consecuencias indeseables del incumplimiento terapéutico en Psiquiatría: Influencia en el pronóstico del paciente esquizofrénico. En F. Puente (Ed.), Adherencia terapéutica: Implicaciones para México (57-59). México, DF: Lepetit.

Ross, H. E., Cutler, M. y Sklar, S. M. (1997). Retention in substance abuse: Role of psychiatric symptom severity. American Journal on Addictions, 6(4), 293-303.

Saucedo, M. (1997). Frecuencia y causas de deserción de los pacientes que asisten a consulta subsecuente de la División de Servicios Clínicos del Instituto Mexicano de Psiquiatría. Salud Mental, 20(10), 13-18

Sidman, M. (1960). Tactics of scientific research. Nueva York: Basic Books.

Siqueland, L., Crits-Christoph, P., Frank, A., Daley, D., Weiss, R., Chittams, J., Blaine, J. y Luborsky, L. (1998). Predictors of dropout from psychosocial treatment of cocaine dependence. Drug and Alcohol Dependence, 52(1), 1-13.

Swanson, A. J., Pantalon, M. V. y Cohen, K. R. (1999). Motivational interviewing and treatment adherence among psychiatric and dually diagnosed patients. Journal of Nervous and Mental Disease, 187(10), 630-635. 
Alejandro Velázquez R., Juan José Sánchez Sosa, Maria del Carmen Lara y Héctor Sentíes

Tehrani, E., Krussel, J., Borg, L. y Munk-Jorgensen, P. (1996). Dropping out of psychiatric treatment: A prospective study of a first admission cohort. Acta Psychiatrica Scandinavica, 94(4), 266-271.

Wiseman, E. J., Henderson, K. L. y Briggs, M. J. (1997). Outcomes of patients in a VA ambulatory detoxification program. Psychiatric Services, 48(2), 200-203.

World Health Organization (1996). Mental health. In: Ottawa Charter for Health Promotion, http://www.who.dk/policy/ottawa.htm.fact sheet N.130, Agosto.

Zimbroff, D. L., Kane, J. M. y Tamminga, C. A. (1998). Sertindole versus haloperidol for schizophrenia. American Journal of Psychiatry, 155(9), 1303-1304. 